\title{
Particle Swarm Optimization Based Approach for Resource Allocation and Scheduling in OFDMA Systems
}

\author{
Chilukuri Kalyana Chakravarthy ${ }^{1}$, Prasad Reddy ${ }^{2}$ \\ ${ }^{1}$ Department of Computer Science and Engineering, Maharaj Vijayaram Gajapathi Raj College of Engineering, \\ Vizianagaram, India \\ ${ }^{2}$ Department of CS\&SE, Andhra University, College of Engineering, Visakhapatnam, India \\ E-mail: kch.chilukuri@gmail.com,prof.prasadreddy@gmail.com \\ Received March 9, 2010; revised April 10, 2010; accepted May 11, 2010
}

\begin{abstract}
Orthogonal Frequency-Division Multiple Access (OFDMA) systems have attracted considerable attention through technologies such as 3GPP Long Term Evolution (LTE) and Worldwide Interoperability for Microwave Access (WiMAX). OFDMA is a flexible multiple-access technique that can accommodate many users with widely varying applications, data rates, and Quality of Service (QoS) requirements. OFDMA has the advantages of handling lower data rates and bursty traffic at a reduced power compared to single-user OFDM or its Time Division Multiple Access (TDMA) or Carrier Sense Multiple Access (CSMA) counterparts. In our work, we propose a Particle Swarm Optimization based resource allocation and scheduling scheme (PSORAS) with improved quality of service for OFDMA Systems. Simulation results indicate a clear reduction in delay compared to the Frequency Division Multiple Access (FDMA) scheme for resource allocation, at almost the same throughput and fairness. This makes our scheme absolutely suitable for handling real time traffic such real time video-on demand.
\end{abstract}

Keywords: OFDMA, Resource Allocation, Scheduling, Quality of Service, Delay

\section{Introduction}

TDMA and FDMA used for distributing subcarriers in OFDM systems form static subcarrier management schemes. While in OFDM-TDMA, one of the users is assigned all the subcarriers for the entire scheduling interval, in the OFDM-FDMA, each user is assigned predetermined number of subcarriers. However, neither of these techniques is time or frequency efficient: TDMA is a time hog and FDMA is a bandwidth hog. OFDMA is a multi-user OFDM that allows multiple access on the same channel (a channel being a group of evenly spaced subcarriers, as discussed above). WiMAX uses OFDMA, extended OFDM, to accommodate many users in the same channel at the same time. In OFDMA, the OFDMA subcarriers are divided into subsets of subcarriers, each subset representing a subchannel (see Figure 1). Dynamic subcarrier allocation schemes which consider the instantaneous channel conditions have been the main area of research interest recently. The resource allocation is usually formulated as a constrained optimization problem, to either 1) minimize the total transmit power with a constraint on the user data rate $[1,2]$ or 2) maximize the total data rate with a constraint on total transmit power
[3-5]. The first objective is appropriate for fixed-rate applications, such as voice, whereas the second is more appropriate for bursty applications, such as data and other IP applications.

In the downlink, a subchannel may be intended for different receivers or groups of receivers; in the uplink, a transmitter may be assigned one or more subchannels. The subcarriers forming one subchannel may be adjacent or not. The standard indicates that the OFDM symbol is divided into logical subchannels to support scalability,

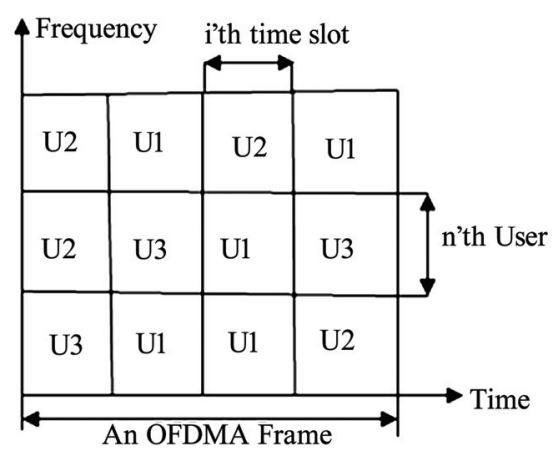

Figure 1. OFDMA frame structure. 
multiple access and advanced antenna array processing capabilities. The multiple access has a new dimension with OFDMA where in a downlink or an uplink user will have a time and a subchannel allocation for each of its communications.

The main motivation for adaptive subcarrier allocation in OFDMA systems is to exploit multiuser Diversity. In a $K$-user system in which the subcarrier of interest experiences i.i.d. Rayleigh fading - that is, each user's channel gain is independent of the others, as the number of users' increases, the probability of getting a large channel gain increases. Further, it was observed that majority of the gain is achieved from only the first few users. Adaptive modulation is the means by which good channels can be exploited to achieve higher data rates.

WiMAX systems use adaptive modulation and coding in order to take advantage of fluctuations in the channel. The basic idea is quite simple: Transmit as high a data rate as possible when the channel is good, and transmit at a lower rate when the channel is poor, in order to avoid excessive dropped packets. While Lower data rates are achieved by using a small constellation, such as QPSK, and low-rate error-correcting codes, such as rate convolutional or turbo codes, the higher data rates are achieved with large constellations, such as 64 QAM, and less robust error correcting codes; for example, rate convolutional, turbo, or LDPC codes. However, a key challenge in AMC is to efficiently control three quantities at once: transmit power, transmit rate (constellation), and the coding rate.

In theory, the best power-control policy from a capacity standpoint is the so-called waterfilling strategy, in which more power is allocated to strong channels and less power allocated to weak channels [6]. In practice, the opposite may be true in some cases. For example, in regions of low gain, the transmitter would be well advised to lower the transmit power, in order to save power and generate less interference to neighboring cells [7].

As mentioned earlier, OFDMA thus facilitates the exploitation of frequency diversity and multiuser diversity to significantly improve the system capacity. In a multiuser System, the optimal solution is not necessarily to assign the best subcarriers seen by a single chosen user since the best subcarrier of one user is also the best subcarrier for another user who has no other good subcarriers. Hence, a different approach should be considered for scheduling the best user. We consider the problem where $\mathrm{K}$ users are involved in the OFDMA system to share $\mathrm{N}$ subcarriers. Each user allocates non overlapping set of subcarriers Sk where the number of subcarriers per user is $\mathrm{J}(\mathrm{k})$. The allocation module of the transmitter assigns subcarriers to each user according to some QoS criteria. QoS metrics in the system are rate and BER. Each user's bit stream is transmitted using the assigned subcarriers and adaptively modulated for the number of bits assigned to the subcarrier. The power level of the modulation is adjusted to meet QoS for given fading of the channel (see Figure 2).

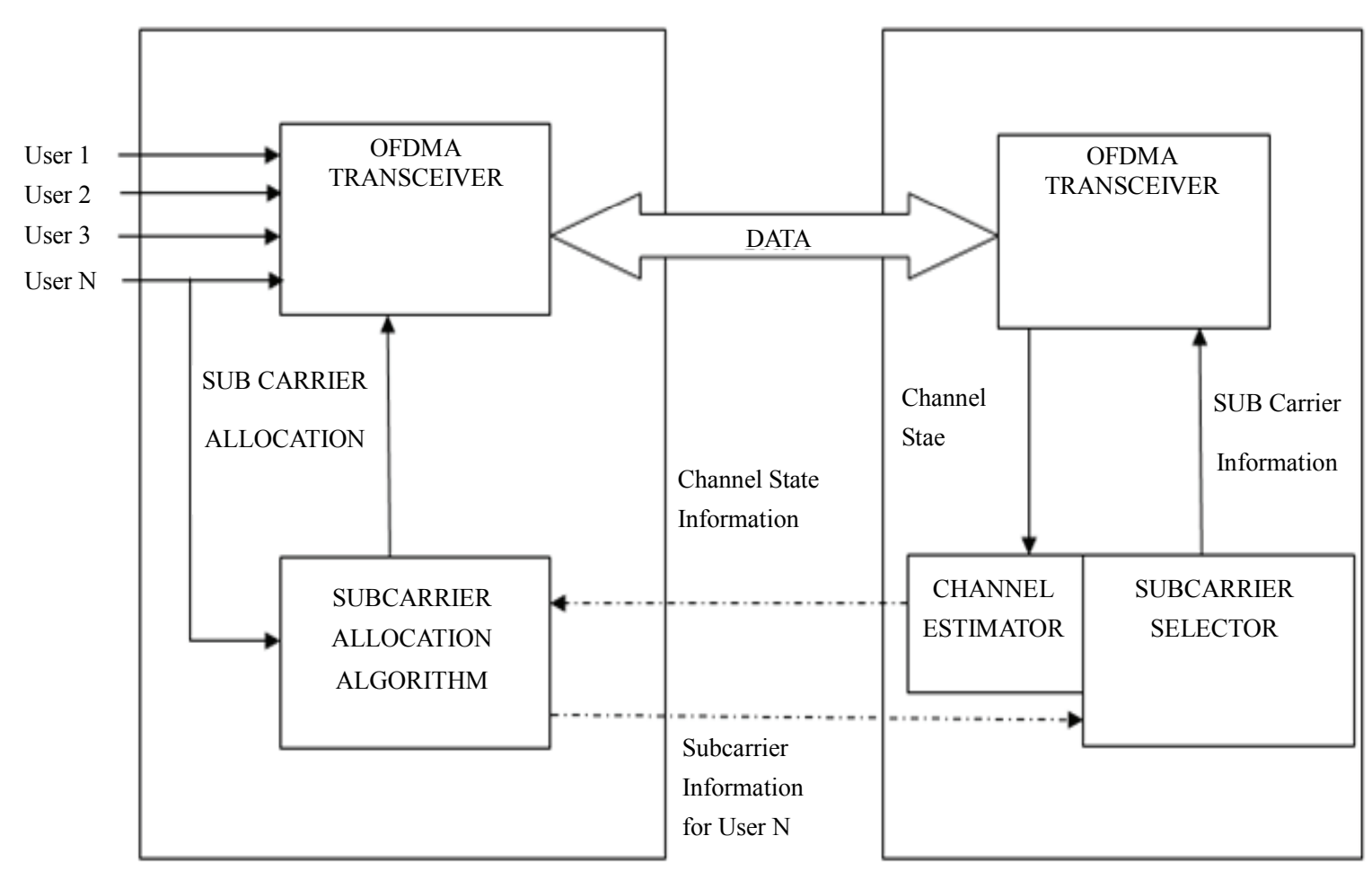

Figure 2. Downlink OFDMA System architecture. 
If $\gamma_{k, n}$ is the indicator of allocating the nth subcarrier to the kth user, the transmission power allocated to the nth subcarrier of $k$ th user can be expressed as $P_{k, n}=f_{k}\left(C_{k, n}, B E R_{k}\right) / \alpha_{k, n}^{2}$ where $f_{k}\left(C_{k, n}\right)$ is the required received power with unity channel gain for reliable reception of $c$ bits per symbol. Therefore, the resource allocation problem with an imposed power constraint can be formulated as

$$
\max C_{k, n}, \gamma_{k, n} R_{k}=\sum_{\mathrm{n}=1}^{\mathrm{N}} C_{k, n} \gamma_{k, n} \text { for all } k
$$

subject to $P_{r}=\sum_{\mathrm{k}=1}^{\mathrm{K}} \sum_{\mathrm{n}=1}^{\mathrm{N}} \frac{f_{k}\left(C_{k, n}, B E R_{k}\right)}{\alpha_{k, n}^{2}} \gamma_{k, n} \leq P_{\max }$

The limit on the total transmission power is expressed as $P_{\max }$ for all $n \in\{1, \ldots, N\}, k \in\{1, \ldots, K\}$ and $C_{k, n} \in\{1, \ldots, M\}$. The proposed method uses the Particle Swarm Optimization for resource allocation and scheduling in a multiuser scenario, considering the rate, power and the subcarrier allocation constraints.

\section{Particle Swarm Optimization}

Particle Swarm Optimization (PSO) is motivated from the simulation of social behavior of animals'. It was introduced by Eberhart \& Kennedy in 1995. In PSO, potential solutions (particles) move dynamically in space. PSO is similar to the other evolutionary algorithms in which the system is initialized with a population of random solutions. A list of Genetic algorithms is given in [8-12]. Each potential solution, call particles, flies in the D-dimensional problem space with a velocity which is dynamically adjusted according to the flying experiences of its own and its colleagues. The location of the ith particle is represented as $X_{i}=\left(x_{i 1}, \ldots, x_{i d}, \ldots, x_{i D}\right)$. The best previous position (which giving the best fitness value) of the ith particle is recorded and represented as $P_{i}=$ $\left(p_{i 1}, \ldots, p_{i d}, \ldots, p_{i D}\right)$, which is also called pbest. The index of the best pbest among all the particles is represented by the symbol g. The location Pg is also called gbest. The velocity for the ith particle is represented as $V_{i}=\left(v_{i 1}, \ldots, v_{i d}, \ldots, v_{i D}\right)$. The particle swarm optimization concept consists of, at each time step, changing the velocity and location of each particle toward its pbest and gbest locations. The particle swarm optimization concept consists of, at each time step, changing the velocity and location of each particle toward its pbest and gbest locations according to the equations $v_{i d}=\mathrm{w} \times v_{i d}+\mathrm{c} 1 \times \operatorname{rand}() \times$ $\left(p_{i d}-x_{i d}\right)+\mathrm{c} 2 \times \operatorname{rand}() \times\left(p_{g d}-x_{i d}\right)$ and $x_{i d}=x_{i d}+v_{i d}$ respectively.where $\mathrm{w}$ is inertia weight, $\mathrm{c} 1$ and $\mathrm{c} 2$ are acceleration constants [13] which is responsible for keeping the particle moving in the same direction, and rand() is a random function in the range $[0,1]$. For the first equation, the first part represents the inertia of pervious velocity; the second part is the "cognition" part, which represents the private thinking by itself which causes the particle to move to regions of higher fitness; the third part is the "social" part, which represents the cooperation among the particles [14]. Thus the social component causes the particle to move to the best region the swarm has found so far.

The PSO algorithm consists of just three steps, which are repeated until some stopping condition is met [15]:

1) Evaluate the fitness of each particle

2) Update individual and global best fitnesses and positions

3) Update velocity and position of each particle

Further, velocity clamping is used to prevent the particle to move too much away from the search space, the limits being confined to $\left[-V_{\max }, V_{\max }\right]$ if the search space spans from $\left[-P_{\max }, P_{\max }\right][16]$.

\section{The Proposed System}

In this work, we propose a Particle Swarm Optimization (PSO) Approach combined with Credit based scheduling to guarantee QOS in WiMAX.

Formal definition of our scheduling model:

$$
\text { Minimize } Z_{k}=\left(\sum_{1 \leq k \leq K}\left|\frac{\sum_{\mathrm{i}=1}^{N} r_{i} x_{i k}}{x_{i k}}-D\right|^{p}\right)^{1 / P}
$$

Subject to $\sum_{i=1}^{N} t_{i} x_{i k} \leq u, 1 \leq k \leq K$

and

$$
\sum_{i=1}^{N} p_{i j} x_{i k} \leq m_{j}, 1 \leq j \leq M, 1 \leq k \leq K ;
$$

where terms 1 and 2 refer to the time and power constraints during scheduling respectively.

$x_{i k}$ are decision variables, where $1 \leq i \leq N$ and $1 \leq k \leq$ $K, x_{i k}$ is 1 if a subcarrier has been allocated, 0 otherwise. The decision of whether to grant the subchannel to the subcarrier is based on whether the subcarrier lies within the range of existing subcarriers for a subchannel. A criteria such as rejection of the subcarrier if it is directly adjacent to a previously allocated subcarrier within the same subchannel and acceptance if not so is used. This results in an improvement of SINR. $N$ is the total number of subcarriers per user and $K$ total number of users, $r_{i}$ is the rate of each allocated subcarrier, $D$ is the target rate for each user, $t_{i}$ is the allocation time for the subcarrier, $u$ is the allowable deadline for a user, $p_{i}$ is the power allocation for each subcarrier, $\mathrm{m}$ is the maximum power allocation for user. $C_{i}$ is the maximum allowable credits for a user. We define different penalty factors as follows: 
Penalty factor for violating time constraint

$$
\alpha=\sum_{k=1}^{k}\left(\max \left(0, \sum_{i=1}^{N} t_{i} x_{i k}-\mathrm{U}\right)\right)
$$

Penalty factor for violating power constraint

$$
\begin{gathered}
\beta=\sum_{k=1}^{K} \sum_{j=1}^{M}\left(m_{j}-\sum_{i=1}^{N} p_{i j} x_{i k}\right) \\
\gamma=\sum_{k=1}^{K} \max \left(\delta_{\min }-\sum_{i=1}^{N} x_{i k}, 0\right)+\max \left(0, \sum_{i=1}^{N} x_{i k}-\delta_{\max }\right)
\end{gathered}
$$

In addition, in the third equation, we also define a constraint on the user using a large portion of a subcarrier repeatedly because this will deny opportunities to other users over this subchannel. We refer to such users as 'selfish users'. We initially assign some credits $\delta_{k}$ to each user $k$, which are incremented when each user gains additional subcarriers and decremented when the user loses them. We define credit thresholds $\delta_{\min }$ and $\delta_{\max }$ such that $\delta_{\min } \leq \delta_{k} \leq \delta_{\max }$ and $\gamma$ is the penalty factor for violating the credit usage.

The fitness function of each user can be evaluated as: Minimize $\mathrm{H}(\mathrm{x})=\mathrm{Z}_{\mathrm{k}}+\mathrm{w}_{1} \alpha+\mathrm{w}_{2} \beta+\mathrm{w}_{3} \gamma$, where $\mathrm{w}_{1}$, $\mathrm{w}_{2}, \mathrm{w}_{3}$ denote the weights for the penalty terms. For generation of the initial swarm, the particle gives more preference to items that have a closer rate to the target rate.

The mapping of the velocities to the probabilities can be carried out by the sigmoid function $S\left(V_{i j}\right)=1 /\left(1+\mathrm{e}^{-v_{i j}}\right)$ where positive velocities drive the bit towards 1 value while negative velocities towards the 0 bit values (see Figure 3).

The particle generation is based on the selection rule $\left|r_{i}-D\right|$ is minimum subject to $S\left(v_{i j}\right)=1$. Hence it gives more selection probability to users that have closer rate to the target rate and are represented by particles with positive velocities (see Figure 4).

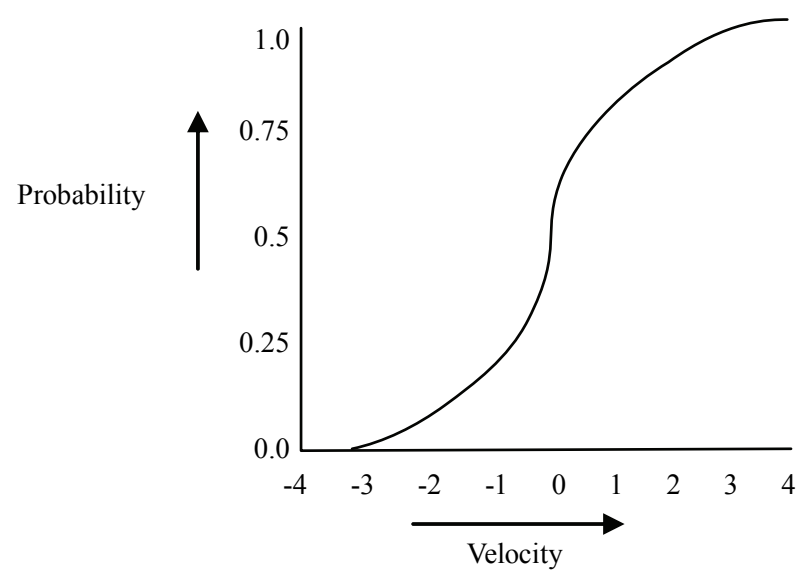

Figure 3. Sigmoid function for probability-velocity mapping.

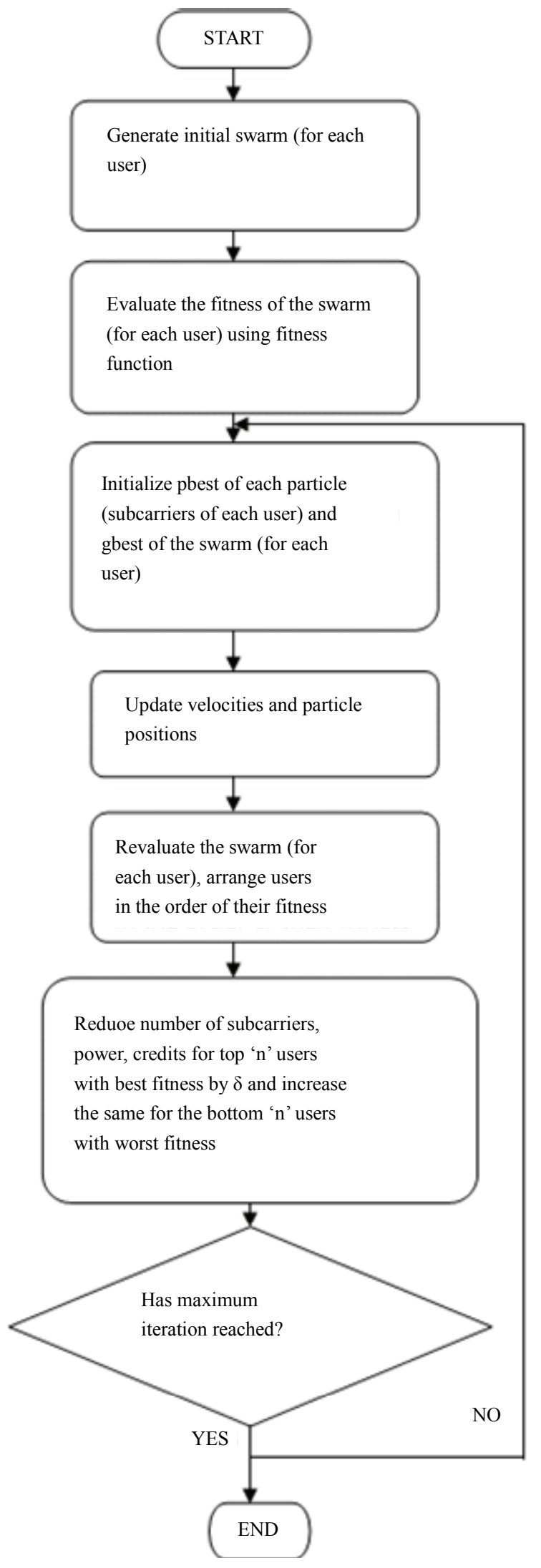

Figure 4. Subcarrier allocation and scheduling in PSORAS. 


\section{The Simulation Model and Results}

We consider the downlink of an OFDMA system with $\mathrm{N}$ subchannels and $\mathrm{K}$ users. The time axis is divided into frames. A frame is further divided into $\mathrm{S}$ time slots, each of which may contain one or several OFDM symbols. The duration of a frame is set to be $5 \mathrm{~ms}$, thus we can assume that the channel quality remains constant within a frame, but may vary from frame to frame. In our simulation, there are 1024 subcarriers, 1 to 50 users in the IEEE 802.16 OFDMA system. Each user transmits 80 bits in an OFDMA symbol. The modulation type in the OFDMA system is confined to QPSK, 16-QAM, 64-QAM. (see Figures 5-7)

Following are the simulation results for the variation in average throughput, fairness index and the average delay with the number of users. The results clearly indicate a reduction in the delay with the proposed swarm based approach compared to the Naïve allocation of subcarriers, i.e. allocation on availability basis in FDMA without considering variation in channel conditions. The results have been evaluated for different sets of target rates and target powers for the subcarriers and the priorities of users are varied after every $5 \mathrm{~ms}$ based on the

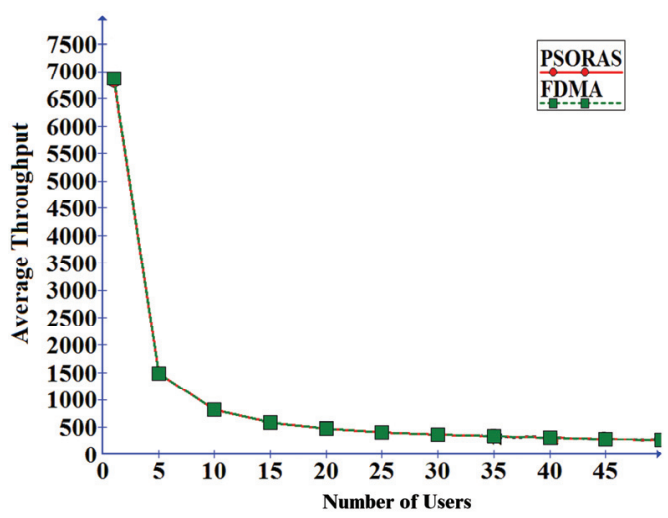

Figure 5. Number of users Vs. Average Throughput.

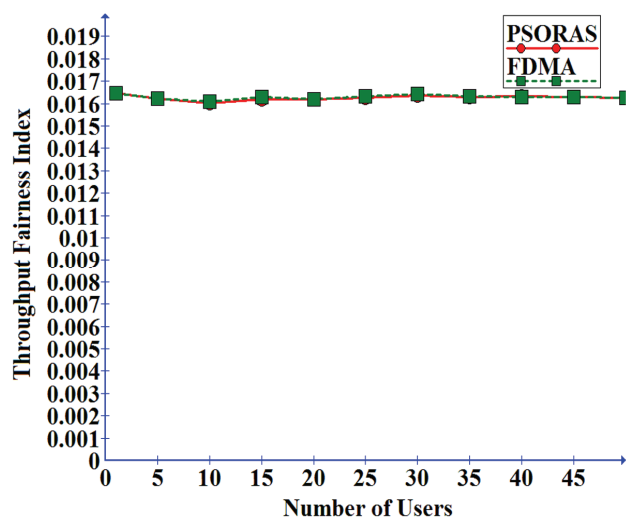

Figure 6. Number of users Vs. Throughput Fairness Index.

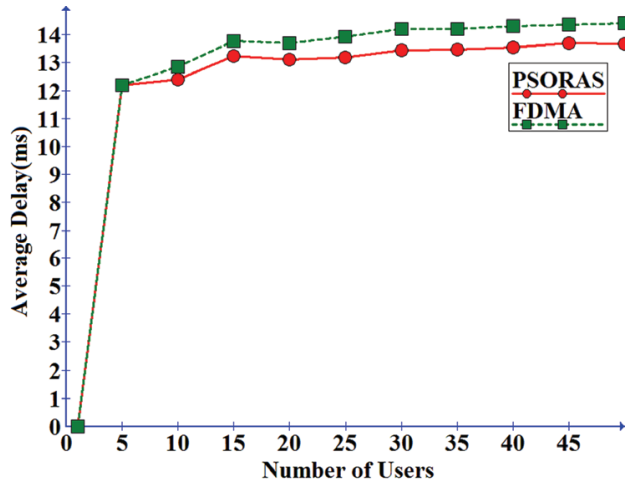

Figure 7. Number of users Vs. Average Delay.

calculated fitness. The throughput fairness index has been calculated as $\tau_{n}=\left(T h_{\max }-T h_{\min }\right) / T h_{\min }$, where $T h_{\min }$ and $T h_{\max }$ are the minimum and maximum values of throughput of each user over ' $n$ ' frames measured in bits.

\section{Conclusions}

Swarm optimization is increasingly finding its place in multiuser downlink MIMO scheduling, smart Antenna array systems etc. In our work, we have proposed a PSO-based fair Resource allocation and scheduling algorithm for the IEEE 802.16 System. We have compared our results with the static FDMA algorithm and have found it offers better delay characteristics with increasing number of users while still maintaining the fairness and throughput utilization. This makes the proposed scheme absolutely useful for real-time applications.

\section{References}

[1] D. Kivanc, G. Li and H. Liu, "Computationally Efficient Bandwidth Allocation and Power Control for OFDMA," IEEE Transactions on Wireless Communications, Vol. 2, No. 6, 2003, pp. 1150-1158.

[2] C. Wong, R. Cheng, K. Letaief and R. Murch, "Multiuser OFDM with Adaptive Subcarrier, Bit, and Power Allocation," IEEE Journal on Selected Areas in Communications, Vol. 17, No. 10, 1999, pp. 1747-1758.

[3] J. Jang and K. Lee, "Transmit Power Adaptation for Multiuser OFDM Systems," IEEE Journal on Selected Areas in Communications, Vol. 21, No. 2, 2003, pp. 171-178.

[4] G. Li and H. Liu, "On the Optimality of the OFDMA Network," IEEE Communications Letters, Vol. 9, No. 5, 2005, pp. 438-440.

[5] C. Mohanram and S. Bhashyam, "A Sub-optimal Joint Subcarrier and Power Aallocation Algorithm for Multiuser OFDM," IEEE Communications Letters, Vol. 9, No. 8, 2005, pp. 685-687. 
[6] G. Manimaran and C. Siva Ram Murthy, "A Fault-Tolerant Dynamic Scheduling Algorithm for Multiprocessor Real-Time Systems and Its Analysis," IEEE Transactions on Parallel and Distributed Systems, Vol. 9, No. 11, 1998, pp. 1137-1152.

[7] R. Chen, J. G. Andrews, R. W. Heath and A. Ghosh, "Uplink Power Control in Multi-Cell Spatial Multiplexing Wireless Systems," IEEE Transactions on Wireless Communications, Vol. 6, No. 7, 2007, pp. 2700-2711.

[8] A. J. Page and T. J. Naughton, "Framework for Task Scheduling in Heterogeneous Distributed Computing Using Genetic Algorithms," 15th Artificial Intelligence and Cognitive Science Conference, Ireland, 2004, pp. 137-146.

[9] A. J. Page and T. J. Naughton, "Dynamic Task Scheduling Using Genetic Algorithms for Heterogeneous Distributed Computing," Proceedings of the $19^{\text {th }}$ IEEE/ACM International Parallel and Distributed Processing Symposium, Denver, 2005, pp. 1530-2075.

[10] A. S. Wu, H. Yu, S. Jin, K.-C. Lin and G. Schiavone, “An Incremental Genetic Algorithm Approach to Multiprocessor Scheduling," IEEE Transactions on Parallel and
Distributed Systems, Vol. 15, No. 9, 2004, pp. 824-834.

[11] A. Y. Zomaya and Y.-H. Teh, "Observations on Using Genetic Algorithms for Dynamic Load-Balancing," IEEE Transactions on Parallel and Distributed Systems, Vol. 12, No. 9, 2001, pp. 899-911.

[12] E. S. H. Hou, N. Ansari and H. Ren, "A Genetic Algorithm for Multiprocessor Scheduling," IEEE Transactions on Parallel and Distributed Systems, Vol. 5, No. 2, 1994, pp. 113-120.

[13] R. Eberhart and Y. Shi, "Particle Swarm Optimization: Developments, Applications and Resources," IEEE International Conference on Evolutionary Computation, Seoul, 2001, pp. 81-86.

[14] J. Kennedy, "The Particle Swarm: Social Adaptation of Knowledge," IEEE International Conference on Evolutionary Computation, Indianapolis, 1997, pp. 303-308.

[15] F. van den Bergh, "An Analysis of Particle Swarm Optimizers," PhD Thesis, University of Pretoria, 2001.

[16] J. Blondin, "Particle Swarm Optimization: A Tutorial," September 2009. 